\title{
Self Pressure Freezing of Caulobacter crescentus for the Preparation of
} Frozen Hydrated Sections.

\author{
S. Yakovlev, B. Luef, L.R. Comolli, and K. H. Downing \\ Lawrence Berkeley National Laboratory, Berkeley, CA 94720
}

Preparation of frozen hydrated sections of biological samples such as bacteria typically requires the use of a high pressure freezing system. While such systems are commercially available, use of this type of equipment in the field, such as on-site during environmental microbiology sample collection, is very difficult and is often inconvenient in the lab. In this work we use the "self-pressurized rapid freezing" method for vitrification of Caulobacter cultures to demonstrate its suitability for preparing material for cryo-sectioning. While a similar method has been described earlier for freeze substitution [1] we adopted it for the preparation of frozen hydrated cryosections. Such a method does not require heavy equipment and therefore, is suitable for field work. The method relies on the fact that cooling water below $4^{\circ} \mathrm{C}$ as well as crystallization causes expansion of water (ice). Due to the low compressibility of water (ice) small expansion builds a high pressure inside of a sealed vessel. Using a sealed vessel thus eliminates the need for a high pressure freezing machine.

It is known that freezing of pure water with high pressure freezing machine does not result in vitreous ice [2]. We applied the self pressure freezing technique to freeze pure water. Commercially available copper freezing tubes (Leica) with inner diameter $400 \mu \mathrm{m}$ were used. Tubes were cleaned, sonicated, filled with water and sealed by crimping with pliers. Then the sealed tubes were immediately plunged in liquid ethane, cut and trimmed with a glass/diamond knife in a cryomicrotome at below $-160 \mathrm{C}$. We have observed that the mechanical properties of different parts within the sample vary. The areas close to the tube wall appear homogeneous and cutting results in a smooth shiny surface. We believe, that fast cooling in this area result in smaller crystallite size and cutting with a diamond knife results in amorphization of the surface [2]. In contrast the central part of the sample cools slower which results in bigger crystallites. Cutting of this part typically results in raw surface. The crossection of the tube shown in Figure 1 demonstrates the difference between the ice in the periphery and the center. Thickness of the ice suitable for cutting varies from $10 \mu \mathrm{m}$ to 150 $\mu \mathrm{m}$ depending on the freezing quality that is difficult to control.

We found that freezing Caulobacter culture as described above, without adding a devirtification agent, does not result in homogenous vitreous sections. This result is similar to the freezing on the high pressure machine, which typically requires adding 20 percent dextran [2]. However, for the self pressure adding 10 percent dextran results in ice that may be cut well. We assume that the main reason for this is using liquid ethane for self pressure freezing, while most high pressure machines use liquid nitrogen. Liquid ethane is a better cryogen and results in faster cooling speed. It is difficult to estimate the pressure inside of the tube due to plasticity of copper but it is probably comparable to that at high pressure freezing. A section obtained with 10 percent dextran is shown in Figure 2a. While the ice in this sample is flexible enough for cutting it still has a large fraction of crystalline ice that gives rise to undesirable contrast in a micrograph. Interestingly, this ice becomes vitreous and contrast disappears under electron beam irradiation. Figure $2 b$ shows an irradiated area in the middle of an undamaged section. We can see that some crystallization induced contrast disappeared under irradiation but unfortunately hydrogen bubbles started to appear inside the bacteria. While the bubbling effect may be somewhat reduced by cutting thinner section and reducing dose rate [3] having crystalline ice in the section may negatively affect internal structure of the bacteria. Figure $3 \mathrm{a}$ shows pre-irradiated section prepared with $10 \%$ dextran. Figures $3 \mathrm{~b}$ and $3 \mathrm{c}$ 
show cryosections obtained with 20 and 30 percent dextran. One can see that adding 20 or 30 percent of dextran to the medium suppresses the appearance of crystalline ice. Unfortunately, the high dextran content decreases contrast between medium and protein. Thus we face a tradeoff between the image contrast which is better for low dextran content and crystalline ice-induced artifacts that are unavoidable at low dextran content. There is another problem with using high dextran content. One of the stages of the sample preparation includes centrifuging the bacterium through the dextran solution in order to increase bacteria abundance. Caulobacter has a relatively low density and spinning in 30 percent dextran solution is not effective, which leads to low number of bacteria within the cryosection. In this work we present results that allow one to choose ideal dextran concentration for specific case.

We conclude that self pressure freezing is a powerful technique for freezing Caulobacter and probably other samples such as biofilms and environmental samples. The method allows to obtain a high quality vitreous sample without using special equipment.

\section{References}

[1] J.L.M. Leunissen \& H. Yi, Self-pressurized rapid freezing (SPRF): a novel cryofixation method for specimen preparation in electron microscopy. Journal of Microscopy, 235(1), p 25-35

[2] A. AL-Amoudi, J. Dubochet \& D. Studer, Amorphous solid water produced by cryosectioning of crystalline ice at $113 \mathrm{~K}$. Journal of Microscopy, 207(2), p. 146-153

[3] Yakovlev, S., et al., Specimen thickness dependence of hydrogen evolution during cryo-transmission electron microscopy of hydrated soft materials. Journal of Microscopy, 2009. 236(3): p. 174-179.

[4] Authors are grateful to David Larson for the useful suggestion on the work on this project. This work was supported by Director, Office of Science, Office of Basic Energy Sciences, Materials Sciences and Engineering Division, of the U.S. Department of Energy; Grant Number: DE-AC02-05CH11231

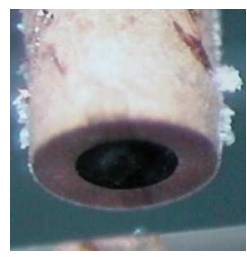

Figure 1: Image of the crossection of copper tube filled with ice. White spot near the center is an area with crystalline ice.
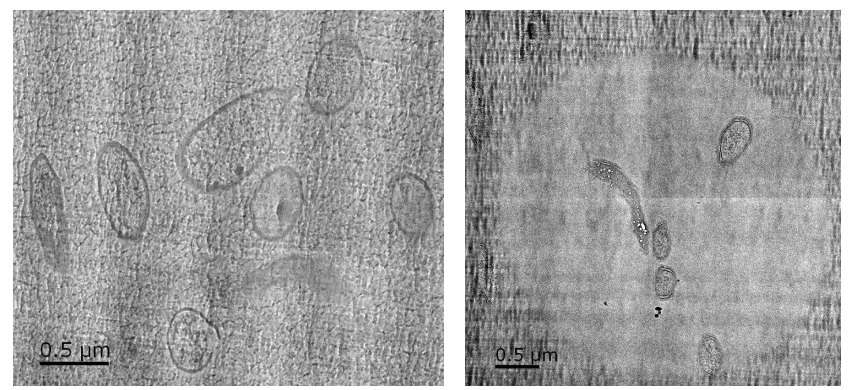

Figure 2: a) Cryosections of Caulobacter culture cushioned through $10 \%$ dextran; b) same cryosection pre-irradiated by electron beam.
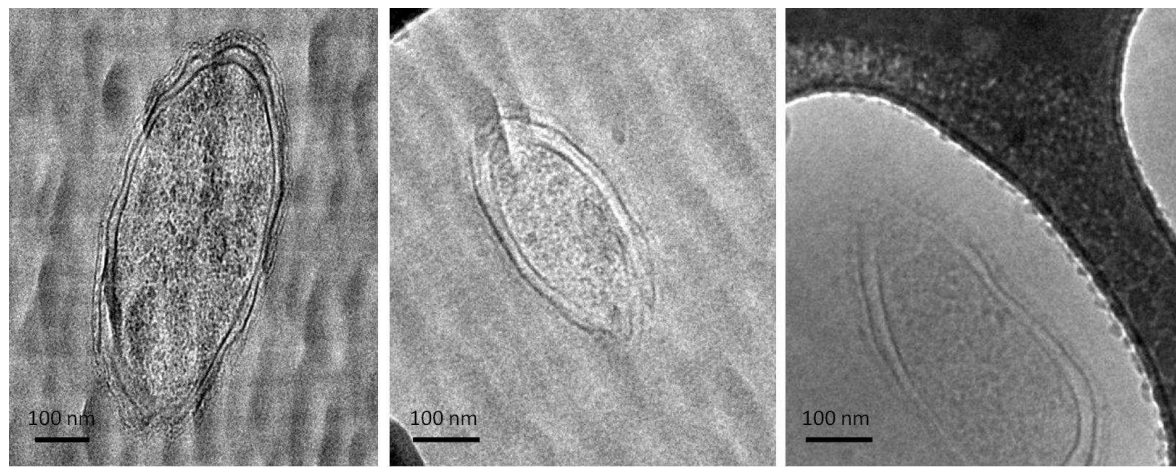

Figure 3: Cryosections of Caulobacter culture cushioned through a) $10 \%$, b) $20 \%$, c) $30 \%$ dextran. 